\title{
Beauty in a smile: the role of medial orbitofrontal cortex in facial attractiveness
}

\author{
J. O’Doherty ${ }^{\mathrm{a}, *}$, J. Winston $^{\mathrm{a}}$, H. Critchley ${ }^{\mathrm{a}}$, D. Perrett ${ }^{\mathrm{b}}$, D.M. Burt ${ }^{\mathrm{b}}$, R.J. Dolan ${ }^{\mathrm{a}, \mathrm{c}}$ \\ ${ }^{a}$ Wellcome Department of Imaging Neuroscience, Institute of Neurology, 12 Queen Square, London WC1 3BG, UK \\ ${ }^{\mathrm{b}}$ School of Psychology, University of St. Andrews, St. Andrews KY16 9JU, UK \\ ${ }^{c}$ Royal Free Hospital School of Medicine, London NW3 2PF, UK
}

\begin{abstract}
The attractiveness of a face is a highly salient social signal, influencing mate choice and other social judgements. In this study, we used event-related functional magnetic resonance imaging (fMRI) to investigate brain regions that respond to attractive faces which manifested either a neutral or mildly happy face expression. Attractive faces produced activation of medial orbitofrontal cortex (OFC), a region involved in representing stimulus-reward value. Responses in this region were further enhanced by a smiling facial expression, suggesting that the reward value of an attractive face as indexed by medial OFC activity is modulated by a perceiver directed smile.
\end{abstract}

(C) 2002 Elsevier Science Ltd. All rights reserved.

Keywords: Facial attractiveness; Face expression; Reward; Orbitofrontal cortex; fMRI

\section{Introduction}

Neuropsychological and functional neuroimaging investigations frequently use face expressions to probe brain regions involved in affect, highlighting regions such as the amygdala, insula and orbitofrontal cortex (OFC) $[2,7,30,43]$. One feature of a face that can elicit a strong affective response in an observer is its attractiveness or beauty. Attractiveness impacts not only on mating success, but also on kinship opportunities, evaluations of personality and performance, as well as employment prospects $[12,13,27,54]$. Indeed a sociobiological perspective on attractiveness suggests it has an evolutionary basis that provides a marker of reproductive fitness [53,54]. Consistent with this is the finding that ratings of facial attractiveness have a high degree of consistency between cultures [40]. Furthermore, preference for attractive faces is found in infants indicating that this phenomenon emerges early in development [28]. Facial attributes that contribute to attractiveness include facial symmetry and the extent to which an individual face conforms to an average prototype $[23,29,37]$. Sexually dimorphic features, which in males corresponds to thick brow ridges and a large jaw structure, and in females corresponds to a small lower face, high cheekbones and thick lips, also contribute to facial attractiveness [39].

\footnotetext{
* Corresponding author. Tel.: +44-207-8337483; fax: +44-207-8131420.

E-mail address: j.odoherty@ fil.ion.ucl.ac.uk (J. O’Doherty).
}

Little is known about how the human brain represents facial attractiveness. An early PET study reported activity of inferior prefrontal cortex during the assessment of facial attractiveness [31]. A recent study has reported an interaction between facial attractiveness and eye-gaze in ventral thalamus extending into striatum, wherein eye-gaze directed at the observer produced enhanced activation in this region relative to the response elicited by faces with eye-gaze averted [25]. This study failed to identify brain regions sensitive to the main effect of attractiveness perhaps reflecting the limited number of face stimuli which were also not optimised to measure responses to attractiveness per se.

The approach taken in the present study is to assume that an attractive face functions as a reward. Evidence from single-cell neurophysiology studies in non-human primates and from neuroimaging studies in humans suggests that one brain region in particular is involved in representing the reward value of stimuli in diverse modalities: the orbitofrontal cortex [45]. OFC is involved in representing the reward value of gustatory, olfactory, auditory and somatosensory stimuli $[8,11,19,34,47]$. This region is also sensitive to abstract reinforcers such as winning or losing money, or positive and negative verbal feedback $[9,16,17,33]$. OFC is also known to be involved in processing at least some facial emotional expressions [7,24].

Consistent with this approach, Aharon et al. [3] explored the hypothesis that attractive faces act as a reward and showed that male subjects were prepared to exert effort by means of key presses in order to gain access to attractive 

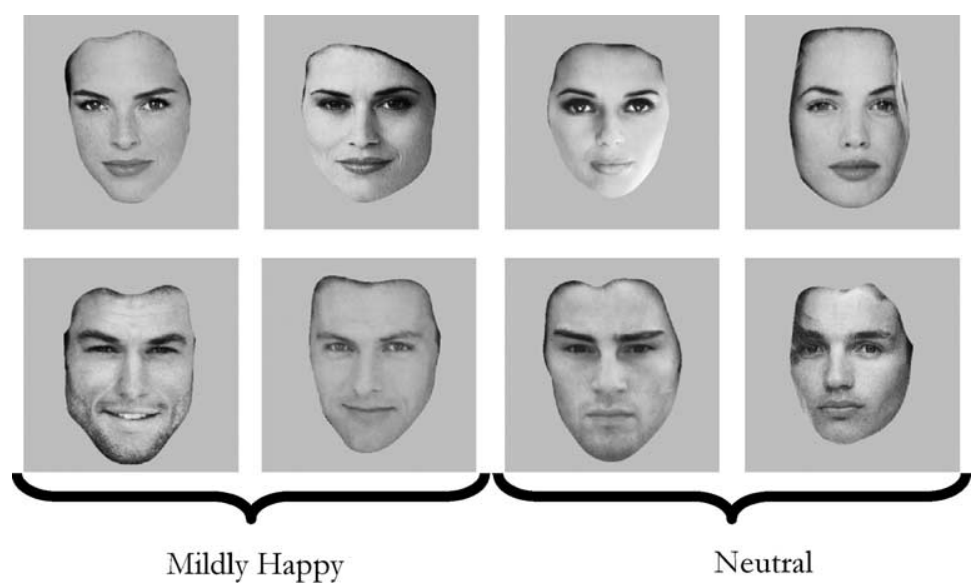

Fig. 1. Examples of face stimuli used in the study. The stimulus set consisted of 12 male and 12 female faces rated as high in attractiveness, and 12 male and 12 female faces rated as low in attractiveness on the basis of prior behavioural ratings. Faces are equated for luminance, eye-gaze is directed towards the observer and the stimuli are approximately balanced across conditions with neutral to mildly happy face expressions. Examples of faces rated by subjects as displaying a happy face expression, are shown on the left of the figure, whereas faces rated by subjects as displaying a neutral face expression are shown on the right. The degree of happiness of faces in the happy condition is quite subtle in comparison to a canonical happy face expression such as that used in the Ekman and Friesen series [14].

female faces indicating that they were prepared to work to obtain views of such faces. In a follow up block design functional magnetic resonance imaging (fMRI) study, Aharon et al. reported activity in the nucleus accumbens in a small group of male subjects to the presentation of attractive female faces, as well as in other brain regions such as OFC in a post-hoc analysis. The use of a block design means that it is not possible to determine whether responses observed were due to the presentation of the attractive faces or to an expectation of reward encompassed within the period in which attractive faces were expected and actually presented.

In the present study, we used an event-related functional magnetic resonance imaging design to measure neural responses while subjects were presented with both male and female faces that were either high or low in attractiveness (see Fig. 1). The use of event-related fMRI enabled us to determine responses directly related to the presentation of the attractive face stimulus without any confound of expectancy as the order of presentation of faces from each condition was fully randomised.

We postulated that facial attractiveness as a reward in the visual modality would evoke event-related responses in orbitofrontal cortex. We also considered a priori that responses to attractive faces could occur in two other brain regions implicated in reward processing, namely the ventral striatum and amygdala $[6,18,50]$. Given the salience of facial attractiveness, we hypothesised that the attractiveness of a face would be processed automatically irrespective of the task performed by the subject, as has already been shown for emotional face expressions [30]. Consequently, the task was for subjects to determine the gender of each face presented in the scanner in the absence of explicit instructions as to the nature of the experimental question until after the scanning was completed. At this point we asked subjects to provide attractiveness ratings for each face. In order to investigate gender-specific responses, we scanned a total of 25 subjects of which 12 were female, allowing a comparison between genders at the random effects level. Furthermore, the fact that faces in the stimulus set displayed either neutral or mildy happy face expressions (expressed as a smile) enabled us to address the issue of the extent to which positive face expression modulates attractiveness responses.

\section{Methods}

\subsection{Subjects}

A total of 25 healthy right-handed normal subjects were included in the experiment of which 13 were male. Twentyfour subjects were Caucasian. The mean age of the male subjects was $24.5 \pm 5.1$ (S.D.), and of the female subjects was $23.1 \pm 3.4$ (S.D.). There were no significant differences in the ages of the subject groups. The subjects were pre-assessed to exclude those with a prior history of neurological or psychiatric illness. All subjects gave informed consent and the study was approved by the local research ethics committee.

\subsection{Stimulus set}

Forty-eight face stimuli were selected from a larger pool of 138 Caucasian faces (depicting healthy normal adults aged between 20 and 35 years) that had been rated by a separate group of subjects $(n=64$ of which 31 were female) for attractiveness in behavioural pilot studies conducted outside the scanner. Attractiveness was rated using a scale from 1 to 7, where 1: 'not attractive' and 7: 'highly attractive'. Twenty-four faces (of which 12 were female) were selected to form the high attractiveness condition and 24 faces (of which 12 were female) were selected to form the 
low attractiveness condition by choosing faces at the high and low ends of the distribution of attractiveness ratings. In addition to attractiveness criteria, faces were chosen to have the following characteristics: eye-gaze forward, head position forwards, and to be approximately balanced across conditions with neutral to mildly happy face expressions. The face images were masked to remove hair and were adjusted to be of approximately equal size and luminance. The faces were centred in a $450 \times 450$ pixel grey background and presented at a screen resolution of $1024 \times 768$ pixels. Examples from the stimulus set are shown in Fig. 1.

\subsection{Experimental paradigm}

Subjects were scanned while being presented with four repetitions of each of the 48 faces in random order, making a total of 192 face presentations. On each trial, a face was presented for $1100 \mathrm{~ms}$ followed by a fixation cross for a further $2000 \mathrm{~ms}$. Randomly interspersed with the face presentation trials were 96 null event trials in which a fixation cross was presented for $3100 \mathrm{~ms}$. Following the presentation of each face subjects were instructed to press one of two buttons on a response key-pad with their right hand to indicate whether the face was of male or female gender. Prior to scanning, subjects were informed that the study was concerned with investigating face processing but no reference was made to the experimental aims. After the scanning task was complete, subjects took part in a self-paced task in which they rated each face for attractiveness and in a separate task using a forced choice procedure, chose from a list of seven emotion categories (neutral, happy, angry, disgust, fear, sad, surprise) which emotion they perceived in each face stimulus (see Section 3). To assist them in the latter task, subjects were provided with a printed sheet depicting each of the seven emotions from the Ekman and Friesen series [14].

\subsection{Imaging procedure}

The functional imaging was conducted by using a 2 Tesla Siemens Vision MRI scanner to acquire gradient echo T2* weighted echo-planar images (EPI) images with blood oxygenation level dependent (BOLD) contrast. Each volume comprised 33 axial slices of $3.3 \mathrm{~mm}$ thickness and $3 \mathrm{~mm}$ in-plane resolution. Volumes were acquired continuously every $2.5 \mathrm{~s}$. These specific parameters produced EPI images in which signal dropout from susceptibility artefact was restricted to far caudal OFC leaving the remaining sectors of OFC intact (see Fig. 3c). Subjects were placed in a light head restraint within the scanner to limit head movement during acquisition. A T1-weighted structural image was also acquired for each subject.

\subsection{Imaging analysis}

The images were analysed using SPM99 (Wellcome Department of Imaging Neuroscience, London, UK). In order to correct for subject motion, the images were realigned to the first volume [20]. The images were then spatially normalized to a standard $\mathrm{T} 2 *$ template with a resampled voxel size of $3 \mathrm{~mm}^{3}$ [20], and spatial smoothing was applied using a gaussian kernel with a full width at half maximum (FWHM) of $10 \mathrm{~mm}$. Intensity normalisation, high pass temporal filtering (using a filter width of twice the minimum inter-trial interval) were also applied to the data. Following pre-processing, statistical analysis was carried out using the general linear model, in which each single event was modelled as a delta function convolved with the haemodynamic response function (HRF) and its temporal derivative. We report here three separate analyses of the data. In the first canonical analysis, the following four events were modelled separately: male high attractive, male low attractive, female high attractive and female low attractive. This enabled the main effects of attractiveness, interactions between-subject gender and attractiveness, and between face gender and attractiveness to be tested by applying linear contrasts of parameter estimates. In the second analysis, subjects' own attractiveness ratings were modelled by creating two regressors of interest: female and male faces, in which the height of the HRF for each stimulus was modulated as a function of the attractiveness rating accorded to that face stimulus. In the third analysis, faces categorised in the happy, intermediate and neutral face expression categories of high and low attractiveness were modelled separately in a canonical analysis to determine the main effect of attractiveness, of happiness and the interaction between attractiveness and happiness. In each of the above analyses, residual effects of head motion were corrected for by including the six estimated motion parameters for each subject as regressors of no interest.

The results from each subject for each of the above analyses were then taken to the random effects level (separately for each analysis) by applying $t$-tests between contrast images to produce group statistical parametric maps. We report results in a priori regions of interest (OFC, striatum and amygdala) at $P<0.001$ uncorrected for multiple comparisons, and indicate if such activations survive corrections for multiple comparisons within a small volume defined around the region of interest using the theory of gaussian random fields [21]. Activations in other brain regions are reported as significant at $P<0.05$ corrected for whole brain. For descriptive purposes, we also report activations outside regions of interest at $P<0.001$ uncorrected but it should be noted that no statistical inference is drawn about such results which clearly require subsequent replication.

\section{Results}

\subsection{Behavioural ratings of attractiveness}

Given the possible effect of subjects' sexual orientation on the imaging results, we asked subjects following scanning to rate their sexual orientation on a 7-point scale from 
1 (exclusively homosexual) to 7 (exclusively heterosexual). Twenty-four subjects rated their orientation as being either 6 or 7 indicating that they had predominantly heterosexual preferences. Subjects also rated the facial attractiveness of each face using a 7-point scale, where $7=$ very attractive, $1=$ not attractive. Faces in the high attractiveness category were rated as being significantly more attractive than the faces in the low attractiveness category as shown by a repeated measures analysis of variance with two within-subject factors: attractiveness and face stimulus gender and one between-subjects factor: subject gender (main effect attractiveness: $F(1,22)=327.121 ; P<0.001)$. A significant interaction was found between-subject gender and attractiveness $(F(1,22)=7.768 ; P<0.05)$, but no significant three-way interaction was found between attractiveness, face stimulus gender and subject gender (across the 24 out of 25 subjects that expressed heterosexual preferences). As can be seen from Fig. 2a, the average attractiveness ratings for the face stimuli in the high attractiveness condition do not overlap with the ratings for the face stimuli in the low attractiveness condition. Average attractiveness ratings are shown for male and female subjects separately in Fig. $2 b$.

\subsection{Neuroimaging results}

\subsubsection{High-low attractive faces}

A linear contrast between the high attractiveness and low attractiveness conditions at the random effects level revealed significant effects in medial OFC $(-3,36,-18, z=4.57$; significant at $P<0.05$ corrected for small volume using a $60 \mathrm{~cm}^{3}$ binary mask defined over the anatomical boundaries of OFC). Significant effects were also observed in bilateral posterior cingulate cortices (left: $9,-48,33, z=5.85$; right: $-6,-45,42, z=4.84$ significant at $P<0.05$ corrected for whole brain volume). Significant effects were also observed in bilateral medial prefrontal cortex $(P<0.001$, uncorrected for multiple comparisons; left: $-6,63,0, z=4.44$; right: $3,42,-12, z=4.39$ ). These results are shown in detail in Fig. 3a.

\subsubsection{Low-high attractive faces}

The opposite contrast was also performed to detect areas with greater responses to low attractive faces relative to high attractive faces. Significant effects $(P<0.05$, corrected for multiple comparisons) were evident in bilateral insula (left: $-33,21,-3, z=5.61$; right: $45,30,9, z=5.59$ ), inferior frontal gyrus $(45,30,9, z=5.59)$ and dorsal para-cingulate cortex $(-6,24,48, z=5.62)$. Effects were also found in right ventrolateral prefrontal cortex bordering OFC at $P<$ 0.001 uncorrected $(45,45,-9, z=3.26)$.

\subsubsection{Interaction between face gender and attractiveness}

In order to determine whether responses to attractive faces were modulated by the gender of the face (opposite sex or same sex), a contrast corresponding to the interaction

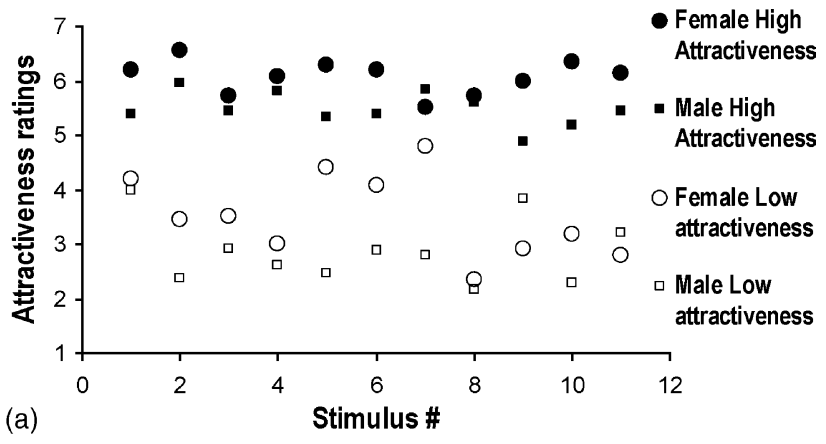

Female subjects
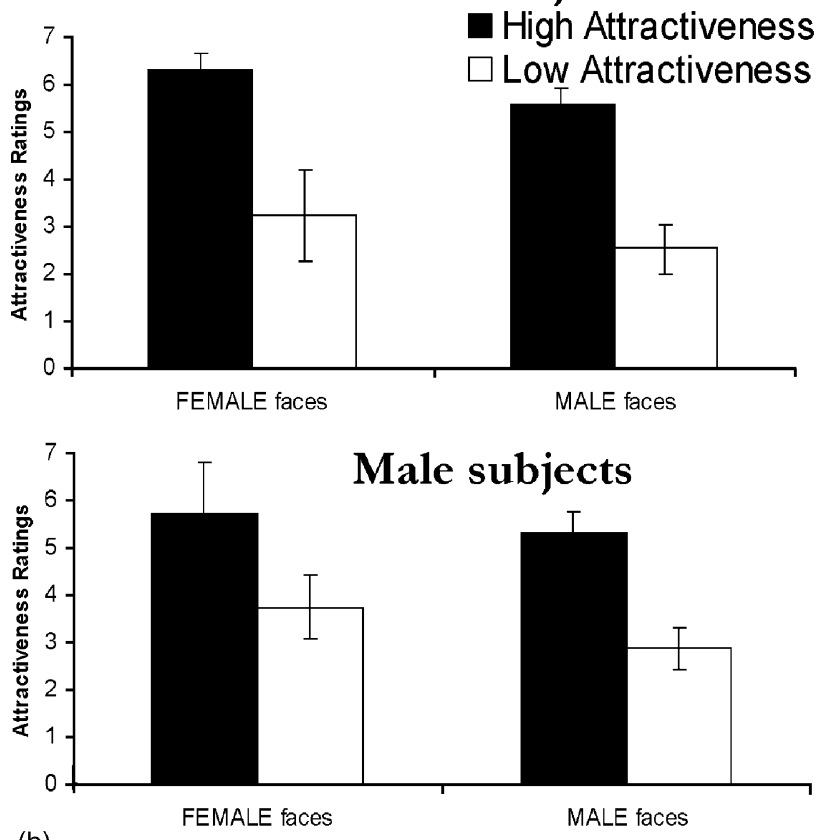

(b)

Fig. 2. (a) Average attractiveness ratings for each of the face stimuli used shown separately for the gender of each face and for the high and low attractiveness conditions. As can be seen, there is no overlap in the attractiveness ratings for stimuli in the high and low attractiveness conditions. (b) Mean attractiveness ratings of the male and female faces in the high and low attractiveness conditions. The ratings for the male and female subjects are shown in separate histograms.

between face gender and attractiveness was performed. No effects were present in regions of interest at $P<0.001$ uncorrected, or in other brain regions at $P<0.05$ corrected for whole brain. Descriptively, we report the sole area in which a significant interaction between face gender and attractiveness was found at $P<0.001$ uncorrected: the anterior ventral bank of the superior temporal sulcus (66, $-30,-9, z=3.59 ; P<0.001$ uncorrected).

\subsubsection{Gender differences in responses to attractive faces}

To test for gender differences in brain regions responding to facial attractiveness a direct comparison was conducted between the linear contrasts of the main effect of attractiveness for each gender at the random effects level. No 
(a)
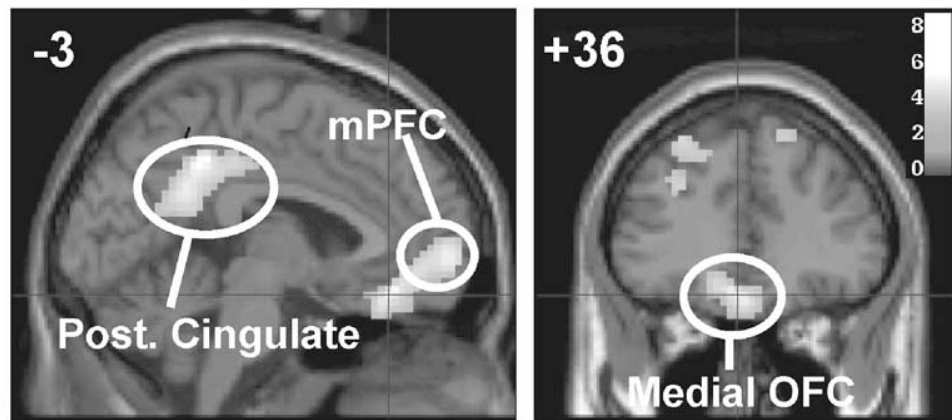

(b)
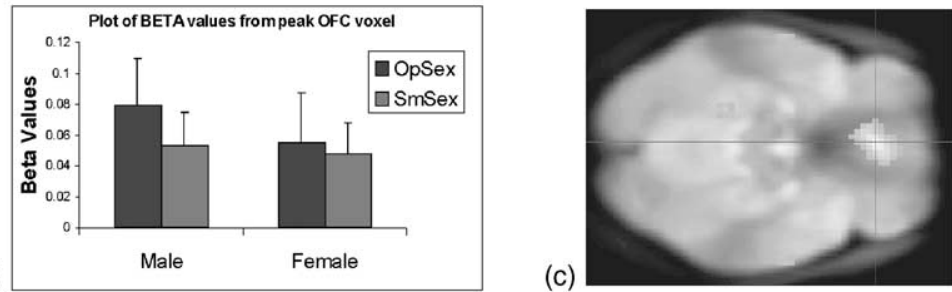

Fig. 3. (a) Main effect of attractiveness. Responses in medial orbitofrontal cortex (OFC), medial prefrontal cortex (mPFC) and posterior cingulate cortex in the main effect of attractiveness shown on coronal and sagittal views, superimposed on a SPM canonical T1 image. The threshold is set at $P<0.001$ uncorrected for illustration. (b) Plot of parameter estimates (beta values) of the effect of attractiveness for a peak voxel in the OFC to the presentation of faces of the opposite or same sex. The averaged beta values are shown separately for male and female subjects. (c) Medial OFC activation shown on a transverse slice superimposed on the averaged EPI image across subjects. This shows that with the imaging parameters used, the region of OFC prone to signal dropout owing to susceptibility artefact is caudal to the area in which activation is reported (see Section 2).

significant gender differences in the responses to the main effect of attractiveness were observed in regions of interest at $P<0.001$ uncorrected or in other brain regions at $P<$ 0.05 corrected. To determine whether any gender-specific responses occur to faces of the opposite sex, a gender comparison was performed for the effect of attractive faces of the opposite sex. A region of medial prefrontal cortex was found to show significantly greater responses to attractive faces of the opposite sex in male subjects than in female subjects $(18,42,0, z=4.98 ; P<0.05$ corrected for whole brain). Descriptively, we report regions responding more in female subjects than in male subjects to attractive faces of the opposite sex at $P<0.001$ uncorrected which were: right inferior temporal gyrus $(48,-60,-27, z=3.61)$ and anatomically related posterior visual areas (including left middle occipital gyrus: $-51,-75,0, z=4.5$ ).

\subsubsection{Parametric analysis of attractiveness responses using the subject's own ratings}

In addition to the canonical analysis, we used the attractiveness ratings provided by each subject as a parametric covariate and tested for areas in which the magnitude of the event-related response followed the profile of the subjects' own attractiveness ratings. The results were similar to those of the canonical analysis of the main effect of attractiveness, with parametric responses in medial orbitofrontal, medial prefrontal and posterior cingulate cortices were all positively correlated with attractiveness ratings $(P<0.001$, uncorrected). The contrast to determine areas that were negatively correlated with the subjects own ratings revealed similar results to the analogous canonical analysis, except that in this case effects were more clearly evident in lateral OFC (48, $42,-15, z=3.67 ; P<0.001$ uncorrected), as well as in adjacent ventrolateral PFC. As in the canonical analysis, significant effects were observed in insula, para-cingulate cortex and inferior frontal gyrus $(P<0.05$, corrected). These results are illustrated in Fig. 4.

\subsubsection{Effects of face expression}

After scanning, subjects were asked to decide which emotional face expression was displayed by each face via a seven-way forced choice procedure (using the categories of angry, sad, fearful, happy, surprised, disgusted or neutral). The mean number of faces rated as neutral across subjects was: 22 (out of 48) and as happy: 17 (out of 48). Three or less faces were rated in each of the other categories of fearful, disgusted, sad, angry or surprised, indicating that subjects did not rate these emotions as being prominently represented in the stimulus set (all faces in the stimulus set were selected to have neutral to mildly happy face expressions). However, 10 faces on average (across subjects) were rated as happy in the high attractiveness conditions and 7 stimuli were rated as happy in the low attractiveness conditions, a difference between the conditions in the number of faces rated as happy that was significant (at $P<0.001$ ).

Given the significant difference in the number of expressions rated as happy between the high and low attractiveness conditions, a further analysis was performed to determine the degree to which a positive face expression modulated attractiveness responses. To achieve this, faces in each condition were categorised by the proportion of subjects who described a particular face as being happy. If over $66 \%$ of 
(a)
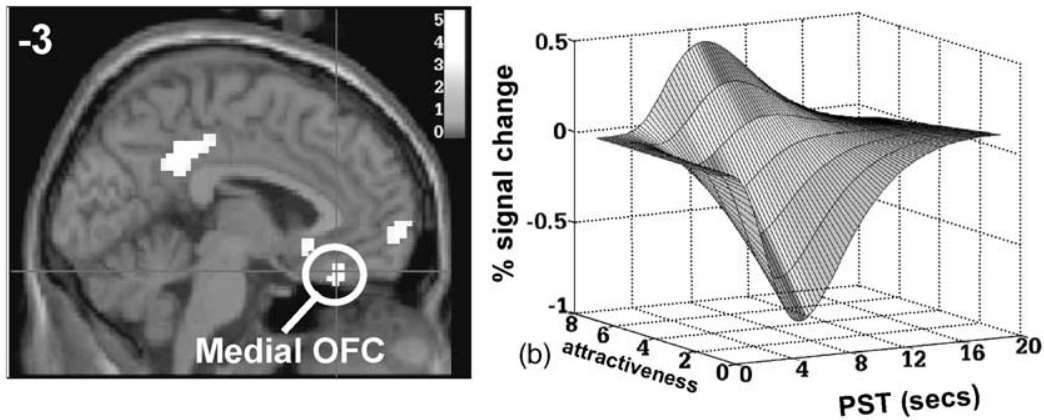

(c)
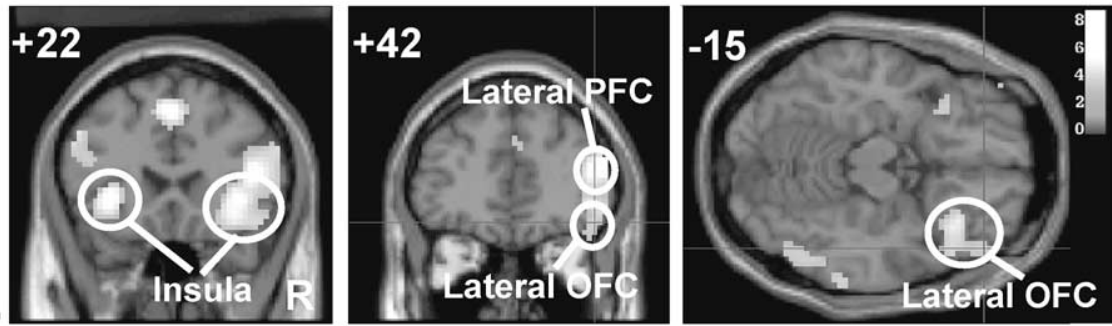

Fig. 4. Results of parametric analysis, in which the subject's own attractiveness ratings were used as regressors. (a) Group random effects result indicating areas positively correlated with the subject's own attractiveness ratings. Responses are illustrated in medial OFC, medial PFC and posterior cingulate cortex and in a fusiform region at a threshold of $P<0.001$ uncorrected for display. (b) A plot of peri-stimulus time (PST) against the parametric responses from a peak voxel in OFC is shown for a single subject. (c) Areas negatively correlated with the subject's own attractiveness ratings (i.e. which demonstrate increased responses to faces that are rated as low in attractiveness). Responses are illustrated in bilateral insula, right lateral OFC and lateral prefrontal cortex.

the subjects rated a face as happy then it was deemed to be happy, and if less than $33 \%$ of subjects rated a face as happy it was deemed to be neutral, with all other faces being placed in an intermediate category. The mean number of subjects rating a face as happy in the happy condition was 19.5 (out of a total of 25), whereas the mean number of subjects rating a face as happy in the neutral condition was 1.3. It should be emphasised that given the stimulus set used, faces in the happy condition display a mild degree of happiness when contrasted with a canonical happy expression as used (a)

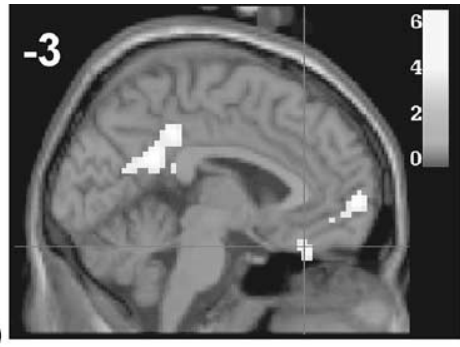

(c)

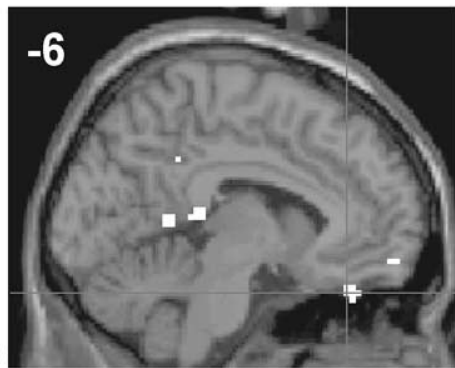

(b)
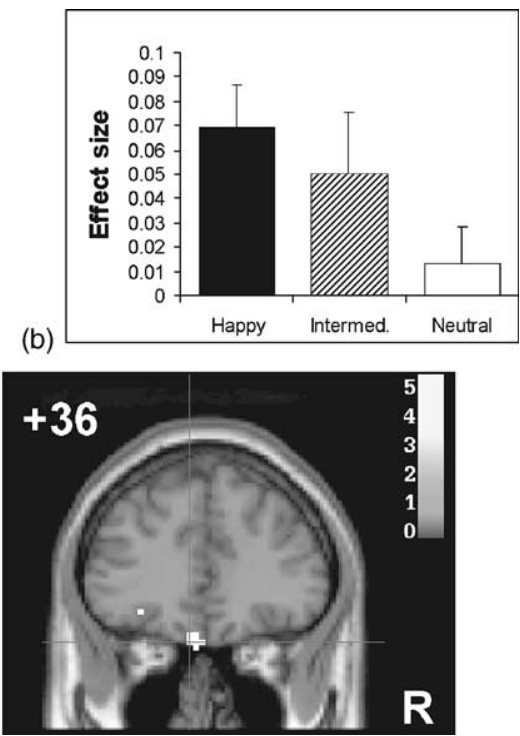

Fig. 5. (a) Results of main effect of attractiveness in $2 \times 2$ factorial model with degree of happy face expression as the other factor (as determined by the proportion of subjects rating a face as happy). (b) Plot of the effect of attractiveness for a peak voxel in the OFC. The values shown corresponds to the mean effect size (parameter estimates of high-low attractive faces) partioned according to the proportion of subjects rating a face as happy. A face was included in the happy condition if $>66 \%$ rated the face as happy, and in the neutral condition if $<33 \%$ rated the face as happy. (c) Results of the interaction between attractiveness and happiness shown for a sagittal and coronal view through medial OFC illustrating that this region responds more to attractive faces that are happy than to attractive faces that are not happy. 
by Ekman and Friesen [14] (see Fig. 1). A one-way analysis of variance test between the mean attractiveness ratings in the three face expression conditions (happy, intermediate and neutral) did not reveal any significant differences in attractiveness ratings between the conditions indicating that happy faces were not rated on average as more attractive.

A factorial model was fitted to the data at the first level in which happy, intermediate and neutral faces in the high and low attractiveness conditions were modelled separately so that the main effect of attractiveness could be tested while taking into account the effects of happiness. At the random effects level, the main effect of attractiveness still yielded significant effects in medial orbitofrontal cortex (as well as medial prefrontal cortex) at $P<0.05$ corrected for small volume and at $P<0.001$ uncorrected, respectively (Fig. 5a). The opposite contrast to determine regions responding more to low than high attractiveness faces once again yielded significant responses in lateral OFC, lateral PFC, insula and para-cingulate cortex. The main effect of happiness did not produce any significant effects in OFC (as assessed by a contrast between happy and neutral faces). A plot of the effect of attractiveness shown separately for faces from the happy, intermediate and neutral expression conditions is shown for a peak OFC voxel in Fig. 5b. This plot reveals that the main effect of attractiveness in OFC is modulated by the extent to which subjects judged a face to be displaying a happy face expression. This effect was tested for more formally by the interaction between happiness and attractiveness, which revealed significant effects in medial $\mathrm{OFC}$ at $P<0.001$ uncorrected $(-6,36,-27, z=3.8$; see Fig. $5 \mathrm{c})$.

\section{Discussion}

The main finding in this paper is that human medial orbitofrontal cortex, a region known to be involved in representing stimulus-reward value, responds to attractiveness or beauty in a face. Furthermore, this region is engaged automatically by attractive faces, even when subjects are performing an unrelated face processing task that does not explicitly assess facial attractiveness. Attractive faces can be considered a rewarding stimulus, and as such engages a brain region known to be involved in processing other kinds of natural rewards such as food, odours or tastes as well as abstract rewards, such as pleasant music or monetary gain $[8,11,33,47]$. Moreover, the degree of activation in orbitofrontal cortex to attractive faces was modulated by the extent to which subjects perceived the presence of a mildly positive face expression, manifest as a smile. Attractive faces that were more consistently rated by subjects as displaying a happy expression, produced stronger responses in the region compared to faces that were displaying a neutral expression, as shown by the significant interaction between attractiveness and happiness (Fig. $5 b$ and c).

It should be noted that the effect we demonstrate cannot be ascribed to a difference in attractiveness ratings between happy and non-happy faces as there was no significant difference in attractiveness ratings between these categories in the present study. However, such an effect has been reported in a previous behavioural study which found that smiling faces were evaluated as more attractive than neutral faces in a large cohort of subjects $(n=330)$ [36]. Given that medial orbitofrontal cortex is associated with representing stimulus-reward value, it may be that the reward value of a face is much enhanced when that face is smiling at the observer (even if subtly as is the case with the stimulus set used here). The presence of a smile may provide an important signal that a reward is or is not attainable. On a behavioural level, this increase in reward value may not be reflected solely in attractiveness ratings which might capture more invariant aspects of facial aesthetics. Rather, this change in reward value may be reflected by changes in other affective ratings such as the perceived pleasantness of the face. In future studies, it will be important to measure a range of different affective judgements in addition to attractiveness in order to address this possibility.

It should be noted that face expression is not the only cue that can modulate neuronal responses to facial attractiveness. Other cues, such as eye-gaze may also play a role [25]. Further, the context in which the faces are presented might also impact on attractiveness responses. In the present study, subjects performed an implicit task in which they made gender judgements during scanning. It is an open question as to whether explicit judgements of attractiveness would produce a similar or different pattern of neural responses to attractive faces.

In contrast to the block design of Aharon et al. [3], we did not observe responses in nucleus accumbens to attractive faces. A likely explanation for the different results observed in the two studies is that the nucleus accumbens activity observed by Aharon et al. was due to reward expectation rather than being driven by presentation of attractive faces per se. There is now considerable evidence to suggest that ventral striatum including nucleus accumbens is involved in incentive motivation or reward expectation, rather than being involved in affective responses to reward itself $[6,26,32,50]$. $\mathrm{OFC}$ on the other hand is known to be involved in representing stimulus affective value as well as being involved in reward expectation $[45,49,51]$. Thus, OFC responses in the present study can be attributed to the affective value of the attractive faces themselves and it is likely that responses were not observed in nucleus accumbens as there was no confound of reward expectation.

We noted differential medial and lateral OFC activity, with medial OFC responding more to high attractive faces than low attractive faces, and lateral OFC (in the parametric analysis) as well as lateral PFC responding more to low attractive faces than high attractive faces. This effect is consistent with previous evidence of a dissociation between medial and lateral OFC responses to a different type of reinforcer: abstract monetary reward and punishment [33]. These findings suggest that for at least these two different types of reinforcer, 
rewards and punishments are represented separately in OFC, with rewards being represented in medial OFC and punishments being represented in lateral OFC. This dissociation may not apply for all types of reward, as pleasant gustatory stimuli have been found to activate lateral OFC [32]. However, dissociable responses have been reported in these regions over time as food was eaten to satiety in a meal in which the food changed from being pleasant to aversive [52].

It is known that lesions of human orbitofrontal cortex produce impairments at gambling and reversal learning tasks in which patients must choose from a set of stimuli and flexibly alter their choice of stimulus on the basis of the rewarding and punishing feedback $[5,46]$. These findings suggest that one function of reward and punishment representations in orbitofrontal cortex is to guide behavioural choice. Indeed, it is possible that medial and lateral orbitofrontal and prefrontal cortices play different roles in this function, such that medial orbitofrontal cortex may be involved in signalling that behaviour toward a given stimulus should be maintained following rewarding feedback, whereas lateral orbitofrontal cortex may be concerned with signalling that behaviour toward a given stimulus should be altered following punishing feedback $[15,33,52]$.

We also tested for areas of the brain that responded more to faces of the opposite sex than to faces of the same sex in those subjects that expressed heterosexual preferences. The only region in which a significant face gender $x$ attractiveness interaction was evident was in the anterior ventral bank of the superior temporal sulcus (STS). This region has been suggested to be part of a network of brain regions involved in social cognition $[1,4,10]$. Neurons in STS are sensitive to face stimuli particularly in responding to social signals conveyed by eye-gaze or head direction $[38,41,42]$. The enhanced activity in this region to attractive faces of the opposite sex may reflect the increased social salience of opposite sex attractive faces. An important caveat is that responses in this region did not survive correction for multiple comparisons and further replication is necessary before firm conclusions can be drawn. Although medial OFC did not show a significant interaction, it can be seen from Fig. 3a that there is a non-significant trend in that the size of the response to opposite sex faces is larger than for same sex faces.

We also tested for gender differences in the responses to attractive faces, and more specifically to opposite sex attractive faces. Although no gender differences were found in responses to attractive faces in general, a significant gender difference was observed in the responses to opposite sex attractive faces in right ventromedial prefrontal cortex. This region showed significantly greater responses to opposite sex attractive faces in males than in females. Although the functions of this brain region is poorly understood, damage to this region and adjacent OFC has been found to produce impairments in affective decision making [5]. In human neuroimaging studies this region has been found to respond to monetary reward or during pleasant affective states such as humour [22,33]. It is of interest to note that in non-human primates this region has strong efferent connections with the hypothalamus, an area involved in sexual behaviour that has been implicated in gender-specific responses to sex pheromones in humans $[35,44,48]$.

To conclude, we present evidence that attractive faces produce activation in human orbitofrontal cortex, when subjects are not explicitly assessing faces for attractiveness. The finding of responses in orbitofrontal cortex, a region known to be involved in representing stimulus-reward value is consistent with the hypothesis that attractive faces can be considered to be a type of reward. Responses in these regions are modulated by the extent to which subjects perceive the presence of a positive face expression, indicating that the reward value of an attractive face is enhanced if that face is smiling at the observer.

\section{Acknowledgements}

This work is supported by a programme grant to RJD from the Wellcome Trust.

\section{References}

[1] Adolphs R. The neurobiology of social cognition. Current Opinion in Neurobiology 2001;11:231-9.

[2] Adolphs R, Tranel D, Damasio H, Damasio A. Impaired recognition of emotion in facial expressions following bilateral damage to the human amygdala. Nature 1994;372:662-9.

[3] Aharon I, Etcoff N, Ariely D, Chabris CF, O'Connor E, Breiter HC. Beautiful faces have variable reward value. fMRI behavioral evidence. Neuron 2001;32:537-51.

[4] Allison T, Puce A, McCarthy G. Social perception from visual cues: role of the STS region. Trends in Cognitive Science 2000;4:267-78.

[5] Bechara A, Damasio AR, Damasio H, Anderson SW. Insensitivity to future consequences following damage to human prefrontal cortex. Cognition 1994;50:7-15.

[6] Berridge KC. Food reward: brain substrates of wanting and liking. Neuroscience and Biobehavioral Reviews 1996;20:1-25.

[7] Blair RJ, Morris JS, Frith CD, Perrett DI, Dolan RJ. Dissociable neural responses to facial expressions of sadness and anger. Brain 1999;122:883-93.

[8] Blood AJ, Zatorre RJ, Bermudez P, Evans AC. Emotional responses to pleasant and unpleasant music correlate with activity in paralimbic brain regions. Nature Neuroscience 1999;2:382-7.

[9] Breiter HC, Aharon I, Kahneman D, Dale A, Shizgal P. Functional imaging of neural responses to expectancy and experience of monetary gains and losses. Neuron 2001;30:619-39.

[10] Brothers L. The social brain: a project for integrating primate behaviour and neurophysiology in a new domain. Concepts Neuroscience 1990;1:27-51.

[11] Critchley HD, Rolls ET. Hunger and satiety modify the responses of olfactory and visual neurons in the primate orbitofrontal cortex. Journal of Neurophysiology 1996;75:1673-86.

[12] Dion K, Berscheid E, Walster E. What is beautiful is good. Journal of Personality and Social Psychology 1972;24:285-90.

[13] Dipboye RL, Arvey RD, Terpstra DE. Sex and physical attractiveness of raters and applicants as determinants of resume evaluations. Journal of Applied Psychology 1977;4:288-94.

[14] Ekman P, Friesen WV. Unmasking the face. New Jerssey: Prenctice-Hall; 1975. 
[15] Elliott R, Dolan RJ, Frith CD. Dissociable functions in the medial and lateral orbitofrontal cortex: evidence from human neuroimaging studies. Cerebral Cortex 2000;10:308-17.

[16] Elliott R, Friston KJ, Dolan RJ. Dissociable neural responses in human reward systems. Journal of Neuroscience 2000;20: 6159-65.

[17] Elliott R, Frith CD, Dolan RJ. Differential neural response to positive and negative feedback in planning and guessing tasks. Neuropsychologia 1997;35:1395-404.

[18] Everitt BJ, Parkinson JA, Olmstead MC, Arroyo M, Robledo P, Robbins TW. Associative processes in addiction and reward. The role of amygdala-ventral striatal subsystems. Annals of the New York Academy of Sciences 1999;877:412-38.

[19] Francis S, Rolls ET, Bowtell R, McGlone F, O’Doherty J, Browning A, et al. The representation of the pleasantness of touch in the human brain, and its relation to taste and olfactory areas. NeuroReport 1999;10:453-9.

[20] Friston KJ, Ashburner J, Poline JB, Frith CD, Heather JD, Frackowiak RS. Spatial registration and normalisation of images. Human Brain Mapping 1995;2:165-89.

[21] Friston KJ, Worsley KJ, Frackowiak RS, Mazziotta JC, Evans AC. Assessing the significance of focal activations using their spatial extent. Human Brain Mapping 1994;1:214-20.

[22] Goel V, Dolan RJ. The functional anatomy of humor: segregating cognitive and affective components. Nature Neuroscience 2001;4: 237-8.

[23] Grammer K, Thornhill R. Human (Homo sapiens) facial attractiveness and sexual selection: the role of symmetry and averageness. Journal of Comparative Psychology 1994;108:233-42.

[24] Hornak J, Rolls ET, Wade D. Face and voice expression identification in patients with emotional and behavioural changes following ventral frontal lobe damage. Neuropsychologia 1996;34:247-61.

[25] Kampe KK, Frith CD, Dolan RJ, Frith U. Reward value of attractiveness and gaze. Nature 2001;413:589.

[26] Knutson B, Fong GW, Adams CM, Varner JL, Hommer D. Dissociation of reward anticipation and outcome with event-related fMRI. NeuroReport 2001;12:3683-7.

[27] Landy D, Sigall H. Task evaluation as a function of the performer's physical attractiveness. Journal of Personality and Social Psychology 1974;4:299-304.

[28] Langlois JH, Ritter JM, Roggmann LA, Vaughn LS. Facial diversity and infant preferences for attractive faces. Developmental Psychology 1991;27:79-84.

[29] Langlois JH, Roggmann LA. Attractive faces are only average. Psychological Science 1990;1:115-21.

[30] Morris JS, Frith CD, Perrett DI, Rowland D, Young AW, Calder AJ, et al. A differential neural response in the human amygdala to fearful and happy facial expressions. Nature 1996;383: $812-5$.

[31] Nakamura K, Kawashima R, Nagumo S, Ito K, Sugiura M, Kato $\mathrm{T}$, et al. Neuroanatomical correlates of the assessment of facial attractiveness. NeuroReport 1998;9:753-7.

[32] O'Doherty J, Deichmann R, Crtichley HD, Dolan RJ. Neural responses during anticipation of a primary taste reward. Neuron 2002;33:815-26.

[33] O’Doherty J, Kringelbach ML, Rolls ET, Hornak J, Andrews C. Abstract reward and punishment representations in the human orbitofrontal cortex. Nature Neuroscience 2001;4:95-102.

[34] O'Doherty J, Rolls ET, Francis S, Bowtell R, McGlone F, Kobal G, et al. Sensory-specific satiety related olfactory activation of the human orbitofrontal cortex. NeuroReport 2000;11:399-402.
[35] Oomura Y, Aou S, Koyama Y, Fujita I, Yoshimatsu H. Central control of sexual behavior. Brain Research Bulletin 1988;20:863-70.

[36] Otta E, Folladore AF, Hoshino RL. Reading a smiling face: messages conveyed by various forms of smiling. Perceptual and Motor Skills 1996;82:1111-21.

[37] Penton-Voak IS, Jones BC, Little AC, Baker S, Tiddeman B, Burt $\mathrm{DM}$, et al. Symmetry, sexual dimorphism in facial proportions and male facial attractiveness. Proceedings of the Royal Society of London B 2001;268:1617-23.

[38] Perrett DI, Hietanen JK, Oram MW, Benson PJ. Organization and functions of cells responsive to faces in the temporal cortex. Philosophical Transactions of the Royal Society London 1992;335:23-30.

[39] Perrett DI, Lee KJ, Penton-Voak I, Rowland D, Yoshikawa S, Burt DM, et al. Effects of sexual dimorphism on facial attractiveness. Nature 1998;394:884-7.

[40] Perrett DI, May KA, Yoshikawa S. Facial shape and judgements of female attractiveness. Nature 1994;368:239-42.

[41] Perrett DI, Rolls ET, Caan W. Visual neurones responsive to faces in the monkey temporal cortex. Experimental Brain Research 1982;47:329-42.

[42] Perrett DI, Smith PA, Potter DD, Mistlin AJ, Head AS, Milner $\mathrm{AD}$, et al. Visual cells in the temporal cortex sensitive to face view and gaze direction. Proceedings of the Royal Society of London B 1985;223:293-317.

[43] Phillips ML, Young AW, Senior C, Brammer M, Andrew C, Calder AJ, et al. A specific neural substrate for perceiving facial expressions of disgust. Nature 1997;389:495-8.

[44] Rempel Clower NL, Barbas H. Topographic organization of connections between the hypothalamus and prefrontal cortex in the rhesus monkey. Journal of Comparative Neurology 1998;398:393419.

[45] Rolls ET. The orbitofrontal cortex and reward. Cerebral Cortex 2000;10:284-94.

[46] Rolls ET, Hornak J, Wade D, McGrath J. Emotion-related learning in patients with social and emotional changes associated with frontal lobe damage. Journal of Neurology, Neurosurgery and Psychiatry 1994:57:1518-24.

[47] Rolls ET, Sienkiewicz ZJ, Yaxley S. Hunger modulates the responses to gustatory stimuli of single neurons in the caudolateral orbitofrontal cortex of the macaque monkey. European Journal of Neuroscience 1989;1:53-60.

[48] Savic I, Berglund H, Gulyas B, Roland P. Smelling of odorous sex hormone-like compounds causes sex-differentiated hypothalamic activations in humans. Neuron 2001;31:661-8.

[49] Schoenbaum G, Chiba AA, Gallagher M. Orbitofrontal cortex and basolateral amygdala encode expected outcomes during learning. Nature Neuroscience 1998;1:155-9.

[50] Schultz W, Apicella P, Scarnati E, Ljungberg T. Neuronal activity in monkey ventral striatum related to the expectation of reward. Journal of Neuroscience 1992;12:4595-610.

[51] Schultz W, Tremblay L, Hollerman JR. Reward processing in primate orbitofrontal cortex and basal ganglia. Cerebral Cortex 2000;10: $272-84$.

[52] Small DM, Zatorre RJ, Dagher A, Evans AC, Jones-Gotman M. Changes in brain activity related to eating chocolate: from pleasure to aversion. Brain 2001;124:1720-33.

[53] Symons D. The evolution of human sexuality. Oxford: Oxford University Press; 1979.

[54] Thornhill R, Gangestad SW. Facial attractiveness. Trends in Cognitive Science 1999;3:452-60. 